\title{
Percepciones de las gestantes en torno al cuidado humanizado por enfermería
}

\author{
Ivett Adriana Herrera-Zuleta* \\ Leticia Alexis Bautista-Perdomo** \\ María José López-Reina** \\ Martha Isabel Ordoñez-Correa** \\ Jennifer Johanna Rojas-Rivera** \\ Hicela Suarez-Riascos ${ }^{* *}$ \\ Julián Alberto Vallejo-Moreno**
}

\section{RESUMEN}

Objetivo: determinar las percepciones de comportamiento del cuidado humanizado de enfermería en un grupo de gestantes del servicio de ginecobstetricia de un hospital de alta complejidad del sur-occidente de Colombia, mediante la aplicación de un instrumento, percepciones del cuidado humanizado en enfermería en su primera versión validada por la Universidad Nacional de Colombia. Materiales y Métodos: investigación cuantitativa descriptiva, de corte transversal. La muestra fue de 97 gestantes obtenidos por muestreo no probabilístico por conveniencia, mediante la fórmula para tamaño óptimo cuando la población es conocida. Se tuvo en cuenta las variables sociodemográficas y la percepción de los comportamientos de cuidado humanizado de enfermería. Resultados: el 59 $\%$ de las gestantes percibieron el comportamiento del cuidado humanizado como excelente; las categorías con mayor porcentaje fueron características de la enfermera, priorizar al ser de cuidado y proactividad, mientras que las categorías con menor valor fueron empatía, disponibilidad para la atención y dar apoyo emocional. Conclusión: el cuidado humanizado de enfermería, en todas las categorías, fue calificado como bueno y excelente, lo que demuestra una adecuada percepción por parte de las gestantes; sin embargo, es importante fortalecer aspectos relacionados con la empatía y la actitud.

PALABRAS CLAVES: enfermería, humanización de la atención, maternidad, percepción ${ }^{* * *}$.

Para citar este artículo / To reference this article / Para citar este artigo

Herrera-Zuleta IA, Bautista-Perdomo LA, López-Reina MJ, Ordoñez-Correa MI, Rojas-Rivera JJ, Suarez-Riascos H, et al. Percepciones de las gestantes en torno al cuidado humanizado por enfermería. Rev. cienc. cuidad. 2016; 13(2): 58-72. 


\section{Perceptions of pregnant women regarding the nursing human care}

Objective: determine perceptions of behavior of nursing human care in a group of pregnant women from the obstetrics and gynecology service of a high complexity hospital in the south-west of Colombia, through the application of an instrument, perceptions of nursing human care in its first version, validated by the Universidad Nacional de Colombia. Materials and Methods: crosssectional descriptive quantitative research, the sample was of 97 pregnant women obtained from a non-probabilistic sample by convenience, through the formula for ideal sample size when the population size is known. Social-demographical variables and the perception of behavior of nursing human care were taken in account. Results: $59 \%$ of pregnant women perceived the behavior of nursing human care as excellent; the categories with higher percentage were characteristics of the nurse, prioritize the care of the person and proactivity, while the categories with lower percentage were: empathy, attention availability and emotional support. Conclusion: the nursing human care, in all of its categories, was qualified as good and excellent, which demonstrates an adequate perception by the pregnant women; however, it is important to strengthen aspects related to empathy and attitude.

KEY WORDS: nursing, humanization of assistance, parenting, perception. 


\section{Percepções de mulheres gravidas em torno dos cuidados de enfermagem humanizada}

\section{RESUMO}

Objetivo: determinar as percepções de comportamento do cuidado humanizado de enfermagem em um grupo de mulheres gravidas do serviço de ginecologia e obstetrícia de um hospital de alta complexidade do Colombia, através da aplicação de um instrumento, "Percepções do cuidado humanizado em enfermagem" em sua primeira versão e validado pela Universidad Nacional de Colombia. Materiais e Métodos: pesquisa quantitativa descritiva, de corte transversal. A amostra foi de 97 mulheres gravidas obtidas por amostragem não probabilística por conveniência, através da fórmula para tamanho óptimo quando a população é conhecida. Foram levadas em conta as variáveis sócio demográficas e a percepção dos comportamentos de cuidado de enfermagem humanizada. Resultados: o 59\% das mulheres gravidas perceberam o comportamento do cuidado humanizado como excelente; as categorias com maior porcentagem foram características da enfermeira, priorizar ao ser de cuidado e proatividade, enquanto que as categorias com menor valor foram: empatia, disponibilidade para o atendimento e dar apoio emocional. Conclusão: o cuidado de enfermagem humanizada, em todas as categorias, foi qualificado como bom e excelente, o que demostra uma adequada percepção por parte das mulheres gravidas; no entanto, é importante fortalecer aspectos relacionados com a empatia e a atitude.

PALAVRAS-CHAVE: enfermagem, humanização da assistência, poder familiar, percepção. 


\section{INTRODUCCIÓN}

El cuidado es la esencia de enfermería y está constituido por acciones transpersonales e intersubjetivas para proteger, mejorar y preservar la humanidad, ayudando al sujeto de cuidado a hallar un significado acerca de la enfermedad, sufrimiento, dolor, existencia y, de esta manera, ayudar al otro a adquirir autocontrol, autoconocimiento y autocuración (1-2).

El concepto de cuidado humanizado comprende procedimientos, manejo de protocolos, utilización de habilidades, destrezas y aplicación de conocimiento científico del ejercicio profesional; por tanto, vislumbra más que un buen trato o la búsqueda de la satisfacción del usuario porque, al hacerlo, no sólo el paciente obtiene beneficios, también los recibe el profesional y el equipo que entrega los cuidados (1-3).

El cuidado humanizado está sujeto a multitud de variables relacionadas con el comportamiento tanto del profesional como del usuario, quien necesita de apoyo en un momento crítico de su vida, sin eximir de los cambios generados por el modelo biomédico de la atención, que reduce lo humano a lo biológico (1), dificultando el trabajo del enfermero y promoviendo una actitud regida por normas que empobrece la calidad de la atención (4).

Jean Watson, en su teoría acerca del cuidado humanizado, propone que la relación enfermerapaciente es interpersonal o subjetiva; es ahí donde surge el complemento del cuidado humanizado, el cual implica un esfuerzo mayor de atención y concentración, convirtiéndose en factor indispensable para todas las áreas de desarrollo profesional (5).

En América Latina, durante los años 2010-2013, países como Chile, Brasil, Venezuela y México se han visto aquejados por la reestructuración administrativa de los sistemas de salud y la tecnología (6), los cuales han generado un cuidado basado en lo fisiológico, dejando a un lado al ser humano como un conjunto de mente, cuerpo y espíritu; por lo tanto, en estos países se ha decidido rescatar la importancia de brindar un cuidado de enfermería basado en el aspecto humano, espiritual y transpersonal (7).

En Colombia, el cuidado humanizado aplica conceptos como la relación transpersonal, una explicación del ser, de la conciencia y el cuidado como esencia de la práctica de enfermería, que transporta al paciente hacia la armonía y el equilibrio de su vida, mediante una continua relación de ayuda y confianza entre el paciente y el cuidador, proporcionando conocimiento, respeto y ayuda mutua.

La gestación es el período de mayor vulnerabilidad biológica y psicológica para las mujeres. El apoyo y la presencia en esos momentos de su pareja, un familiar o de otra persona cercana afectivamente contribuye a disminuir el estrés y la ansiedad. Así mismo, la compañía del personal de enfermería como apoyo emocional y facilitador en su relación con el resto del equipo de salud, puede influir en la salud de la gestante (8).

La deshumanización en el cuidado del paciente, especialmente de la gestante, causada por la creciente utilización de las tecnologías, puesto que, si bien se aligera la atención, también se ha llevado a la creación de acciones rutinarias en donde se olvida la importancia del contacto directo.

En un hospital de alta complejidad del sur occidente del país se ha observado que, en los servicios de hospitalización, se generan ambientes estresantes debido a factores como la reforma en los sistemas de salud (9), los problemas administrativos y organizacionales en la prestación de los servicios sanitarios $(1,4)$, la inclusión de la historia clínica sistematizada y manejos de sistemas y tecnologías, generando la pérdida del cuidado humanizado, fenómeno evidenciado en la inconformidad de los usuarios ante su atención.

\section{MATERIALES Y MÉTODOS}

El estudio corresponde a una investigación descriptiva, trasversal y con abordaje cuantitativo. La muestra fue obtenida a través de muestreo no probabilístico por conveniencia, con el promedio mensual de gestantes mayores de edad que ingresaron al servicio de un hospital de alta complejidad del sur occidente de Colombia.

El cálculo del tamaño de la muestra se realizó mediante la fórmula para tamaño óptimo cuando la población es conocida. El índice de confianza aplicado para la fórmula es $95 \%$ equivalente a 1.96 , con un margen de error del $5 \%$, obteniendo los valores de la siguiente manera:

$$
n=\frac{(1,96)^{2}}{130 \cdot(0,5)^{2}+(1,96)^{2} \cdot(0,5 \cdot 0,5)}
$$

ISSN 1794-9831

E-ISSN 2322-7028

Vol. 13 No. 2

Jul - Dic 2016

Cúcuta, Colombia 
Se obtuvo un resultado de 97 gestantes, las cuales fueron incluidas de acuerdo con los siguientes criterios:

- Gestantes mayores de edad.

- Gestantes que lleven como mínimo 24 horas en área de ginecobstetricia del servicio de hospitalización.

La técnica empleada para recolectar la información fue la encuesta auto-dirigida, cuya aplicación comprendió la presentación del cuestionario y la explicación de los ítems que atiende a la interpretación por parte de las encuestadas para el correcto uso del instrumento y validez de las respuestas. Se utilizó el instrumento Percepciones de Cuidado Humanizado de Enfermería (PCHE) Primera Versión, el cual evaluó aspectos sociodemográficos y de percepción del cuidado, elaborado por Rivera (10), validado por Reina (11), con validez de contenido 0.83 y una validez facial de 0.78 .

El instrumento consta de una variable denominada: Percepción de los comportamientos de cuidado humanizado de enfermería, que contiene 31 ítems, los cuales se agrupan, para facilitar el análisis, en nueve categorías que describen la conceptualización del cuidado. Los ítems fueron evaluados a través de la escala de Likert de 4 puntos, que mide la frecuencia de aparición de los comportamientos del cuidado humanizado en el personal de enfermería identificados y los valora así: 1, Nunca; 2, algunas veces; 3, casi siempre; 4, siempre. Para un rango real del instrumento de 31 a 124 puntos por encuesta y la exploración de dos preguntas abiertas que comprenden reconocer las enfermeras encargadas del turno.

Las categorías del instrumento son:

- Dar apoyo emocional: implica conocimientos y sensibilidad sobre los asuntos que más importan a los pacientes. Su objetivo principal es coincidir en la interpretación de lo que es importante y prestar el apoyo adecuado. Clasificación de intervenciones de enfermería definida como la provisión de tranquilidad, resignación y ánimo durante los momentos de estrés, angustia e incertidumbre $(2,12,13)$.

- Sentimientos del paciente: se refiere a las diversas sensaciones, emociones y sentimientos que pueden generarse en el individuo mediante la relación que se da entre enfermera(o)/paciente.

- Empatía: son aquellas actitudes y acciones positivas que surgen en la interacción de cuidado $(10,14)$.

- Apoyo físico: es la manera en que el paciente percibe el comportamiento y actitud de cómo el personal de enfermería, al realizar un procedimiento, al hablarle, se comporta frente a él; cómo brinda comodidad física y mental, cómo atiende sus necesidades y lo hace sentir una mejor persona y no como un simple enfermo.

- Priorizar al ser cuidado: cada paciente es único; por lo tanto, cada persona posee sus propios sentimientos y pensamientos. Por ello, es necesario que el personal de enfermería trate a cada individuo como un ser único y priorice sus respectivos cuidados, atendiendo cada una de sus inquietudes y llamados (15).

- Cualidades del hacer de la enfermera: el profesional de enfermería debe tratar de conocer y comprender factores que estén ocasionando incertidumbre en el paciente, para poderle ofrecer apoyo, reforzarle las defensas, hacerlo sentir protegido y seguro, responder a las preguntas concretas que formule y darle la información precisa y comprensible.

- Características de la enfermera: son las cualidades, actitudes y comportamientos que distinguen a la enfermera(o) en su trato y cuidado con los pacientes. La empatía, la generosidad, la bondad, la cordialidad, una buena comunicación, la atención oportuna, la recursividad, el empeño y la eficiencia hacen parte de algunas de las características esenciales de la enfermería (16).

- Disponibilidad para la atención: es aquella actitud de disposición que tiene el personal de enfermería para identificar el momento oportuno para brindar cuidado a cada paciente, estar pendiente de su sentir, estados de ánimos, su dolor y sus necesidades de cuidado, para actuar en forma oportuna y eficaz (17).

- Proactividad: definida como todos aquellos comportamientos de la enfermera(o) que 
denotan atención anticipada (18). Luego de realizar la recolección de los datos mediante la encuesta autodirigida, éstos se integraron utilizando la codificación de las variables empleadas en el instrumento y las respuestas brindadas por las gestantes.

Para este proceso se utilizaron los programas de análisis de información general y de procesamiento estadístico Microsoft Excel 2013 y SPSS versión 22. Se condensó la información en tablas de distribución de frecuencias y se agruparon los datos respecto a conjuntos de variables que se confrontaron entre sí para encontrar resultados estadísticamente significativos mediante pruebas de significancia como Chi-cuadrado de Pearson y test exacto de Fisher (1920) que, una vez cumplieron los criterios necesarios, permitieron condensar la información para el análisis de la percepción global y en relación con las categorías presentadas del cuidado humanizado brindado por el personal de enfermería desde la perspectiva de los pacientes.

Este proyecto se clasificò, según la Resolución No. 008430 de 1993 (21), en la categoría de investigación sin riesgo, en la cual no se realiza ninguna intervención o modificación intencionada de las variables biológicas, fisiológicas, psicológicas o sociales de los individuos que participaron en el estudio.

El aval para la utilización del instrumento se obtuvo a través de una carta de autorización dada por los autores del instrumento; el aval ético en la institución de alta complejidad donde se realizó el estudio fue dado a través del código 103-1.5.32. El consentimiento informado fue leído y explicado a los pacientes, quienes participaron de forma voluntaria. Se explicó previamente que los datos obtenidos iban a ser utilizados para la descripción de la percepción del cuidado humanizado brindado por el personal de enfermería y que la información sería de carácter confidencial y privado.

\section{OBJETIVOS}

\section{Objetivo general}

Determinar las percepciones de comportamiento del cuidado humanizado de enfermería en un grupo de gestantes del servicio de ginecobstetricia de un hospital de alta complejidad del sur occidente del país

\section{Objetivos específicos}

- Describir las percepciones de las gestantes en relación con las cualidades, características y disponibilidades de las enfermeras en la atención que brindan.

- Identificar cómo perciben las usuarias el cuidado psico-afectivo brindado por el personal de enfermería.

- Identificar las percepciones de las gestantes en relación con el apoyo físico y la prioridad que se le brinda en su cuidado.

- Clasificar la percepción global de las gestantes en relación con el cuidado que brinda el personal de enfermería.

\section{RESULTADOS}

La recolección de la información para el presente estudio se desarrolló en los meses de enero, febrero y marzo de 2015. Las limitaciones como vivencia del estudio ocurrieron con la obtención del universo y muestra y en el retraso del inicio de la recolección de la información debido a que, inicialmente, no se contó con la información estadística de las pacientes ingresadas al servicio, por lo cual se realizó una verificación manual en libros de registro de maternas.

\section{Caracterización sociodemográfica}

En el estudio participaron 97 pacientes gestantes, quienes permanecieron más de 24 horas hospitalizadas en el servicio de ginecobstetricia. Entre los objetivos se tuvo en cuenta, en primera instancia, la caracterización sociodemográfica, que es una variable indispensable para el análisis de las percepciones de cada participante. Dentro de esta variable se encuentran aspectos relevantes como las edades entre 26 a 35 años, con un porcentaje de $44 \%$; la procedencia rural que prevalece sobre la urbana, con un porcentaje de $52 \%$; a nivel educativo, las gestantes se encontraron con secundaria básica en un $33 \%$, seguida de media superior con un $26 \%$ y primaria con un $25 \%$.

En lo relacionado con el estrato socioeconómico, las gestantes pertenecen a los estratos 1 y 2 en un 58 $\%$ y $25 \%$ respectivamente; el tiempo promedio de estadía fue de un día (59\%) y de 2 días (31\%). Las
ISSN 1794-9831

E-ISSN 2322-7028

Vol. 13 No. 2

Jul - Dic 2016

Cúcuta, Colombia 
gestantes reconocen en mayor porcentaje al personal se puede apreciar en la tabla 1.

Tabla 1. Distribución del perfil sociodemográfico de las gestantes del servicio de ginecobstetricia de un hospital de alta complejidad.

\begin{tabular}{|c|c|c|}
\hline CARACTERISTICAS & No. PACIENTES & $\%$ \\
\hline \multicolumn{3}{|l|}{ EDAD } \\
\hline $18-25$ años & 38 & $39 \%$ \\
\hline $26-35$ años & 43 & $44 \%$ \\
\hline Mayores de 35 años & 16 & $16 \%$ \\
\hline \multicolumn{3}{|l|}{ PROCEDENCIA } \\
\hline Rural & 52 & $54 \%$ \\
\hline Urbano & 45 & $46 \%$ \\
\hline \multicolumn{3}{|l|}{ NIVEL EDUCATIVO } \\
\hline Ninguno & 5 & $5 \%$ \\
\hline Primaria & 24 & $25 \%$ \\
\hline Secundaria & 32 & $33 \%$ \\
\hline Media superior & 25 & $26 \%$ \\
\hline Educación superior & 11 & $11 \%$ \\
\hline \multicolumn{3}{|l|}{ ESTRATO } \\
\hline 0 & 6 & $6 \%$ \\
\hline 1 & 59 & $61 \%$ \\
\hline 2 & 25 & $26 \%$ \\
\hline 3 & 7 & $7 \%$ \\
\hline 4 & 0 & $0 \%$ \\
\hline \multicolumn{3}{|l|}{ TIEMPO DE ESTANCIA EN DIAS } \\
\hline 1 & 47 & $49 \%$ \\
\hline 2 & 31 & $32 \%$ \\
\hline 3 & 9 & $9 \%$ \\
\hline 4 & 3 & $3 \%$ \\
\hline 5 & 5 & $5 \%$ \\
\hline 6 & 1 & $1 \%$ \\
\hline \multicolumn{3}{|l|}{ RECONOCE A LA ENFERMERA } \\
\hline $\mathrm{Si}$ & 59 & $61 \%$ \\
\hline No & 38 & $39 \%$ \\
\hline \multicolumn{3}{|l|}{ RECONOCE A LA AUXILIAR } \\
\hline $\mathrm{Si}$ & 70 & $72 \%$ \\
\hline No & 27 & $28 \%$ \\
\hline
\end{tabular}

Fuente: PCHE, 2015. 


\section{Percepción de cuidado humanizado}

El instrumento Percepciones de Cuidado Humanizado (PCHE) se agrupó para su análisis en 9 categorías, cada una de las cuales obtuvo resultados que permitieron tener un panorama global de la percepción que tienen las gestantes evaluando el cuidado humanizado. Los resultados mostraron que el $59 \%$ de las gestantes percibe el cuidado humanizado como excelente, el $33 \%$ como bueno, el $8 \%$ aceptable y el $0 \%$ como deficiente.
Las variables mejores calificadas por las gestantes fueron: características de la enfermera con $74 \%$, seguida de priorizar al ser cuidado con el $71 \%$, proactividad con el $70 \%$ y sentimientos del paciente con el $66 \%$. Dentro de los porcentajes más elevados y calificados como deficientes se encuentran: la empatía con el $10 \%$ y disponibilidad para la atención con el 6 $\%$, lo que hace aún más necesaria la verificación del proceso de cuidado que brindan los enfermeros (as) a sus pacientes. Se puede apreciar la figura 1 para visualizar cada resultado por ítem.

Figura 1. Resultados globales obtenidos en el instrumento PCHE para cada una de las categorías.

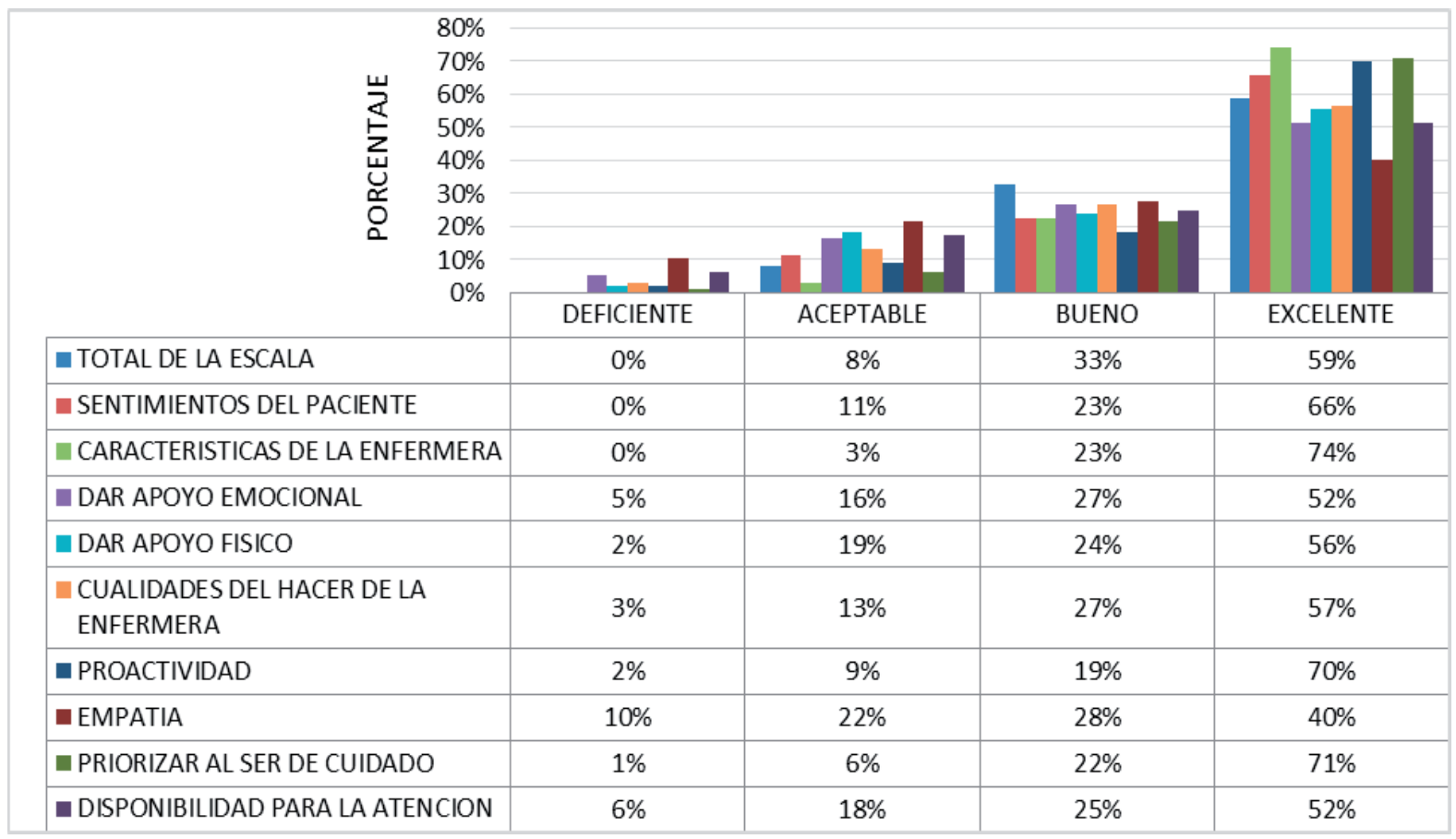

Fuente: PCHE, 2015.

\section{Análisis bivariado de las percepciones obtenidas de las gestantes}

Las variables descritas para el estudio fueron cruzadas para determinar, de acuerdo con los resultados, las asociaciones y significancia entre una y otra. El análisis bivariado se realizó mediante prueba de chi cuadrado de Pearson y test exacto de Fisher, cuyos resultados revelaron que las variables nivel educativo y estrato socioeconómico, al compararlas con las categorías, fueron estadísticamente significativas, con un resultado de $\mathrm{p}<0.05$, para lo cual las percepciones de las gestantes son determinadas de acuerdo al nivel educativo y estrato socioeconómico que presenten. Entre mayor es el nivel educativo, mayor exigencia se tiene en el momento de calificar los ítems. Las variables edad y procedencia no fueron estadísticamente significativas para los ítems desarrollados en las encuestas. La variable reconoce a la enfermera y/o auxiliar de enfermería no arrojó ninguna relevancia para la asociación con los ítems desarrollados. Los resultados se presentan a continuación en la tabla 3.
ISSN 1794-9831

E-ISSN 2322-7028

Vol. 13 No. 2

Jul - Dic 2016

Cúcuta, Colombia 
Tabla 3. Cruce de variables y resultados de pruebas de significancia estadística.

Vol. 13 No. 2

Jul - Dic 2016

Cúcuta, Colombia

\begin{tabular}{|c|c|c|c|c|c|c|c|}
\hline \multicolumn{2}{|c|}{ Variables } & \multirow{2}{*}{$\begin{array}{c}\text { Pearson } \\
0,220\end{array}$} & \multirow{2}{*}{$\begin{array}{l}\text { Fisher } \\
0,199\end{array}$} & \multicolumn{2}{|c|}{ Variables } & \multirow{2}{*}{$\begin{array}{c}\text { Pearson } \\
0,028\end{array}$} & \multirow{2}{*}{$\begin{array}{r}\text { Fisher } \\
0,086\end{array}$} \\
\hline \multirow{10}{*}{$\underset{\tilde{T}}{\tilde{T}}$} & Resultados global & & & \multirow{10}{*}{ 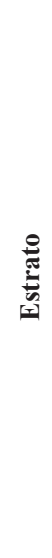 } & Resultados global & & \\
\hline & Categoría Sentimientos del paciente & 0,909 & 0,924 & & Categoría Sentimientos del paciente & 0,083 & 0,086 \\
\hline & $\begin{array}{l}\text { Categoría Características de la } \\
\text { enfermera }\end{array}$ & 0,802 & 0,717 & & $\begin{array}{l}\text { Categoría Características de la } \\
\text { enfermera }\end{array}$ & 0,021 & 0,072 \\
\hline & Categoría Dar apoyo emocional & 0,346 & 0,299 & & Categoría Dar apoyo emocional & 0,311 & 0,228 \\
\hline & Categoría Dar apoyo físico & 0,513 & 0,622 & & Categoría Dar apoyo físico & 0,223 & 0,253 \\
\hline & $\begin{array}{l}\text { Categoría Cualidades del hacer de la } \\
\text { enfermera }\end{array}$ & 0,178 & 0,224 & & $\begin{array}{l}\text { Categoría Cualidades del hacer de la } \\
\text { enfermera }\end{array}$ & 0,425 & 0,330 \\
\hline & Categoría Proactividad & 0,219 & 0,195 & & Categoría Proactividad & 0,458 & 0,461 \\
\hline & Categoría Empatía & 0,708 & 0,670 & & Categoría Empatía & 0,640 & 0,645 \\
\hline & Categoría Priorizar al ser de cuidado & 0,068 & 0,069 & & Categoría Priorizar al ser de cuidado & 0,275 & 0,308 \\
\hline & $\begin{array}{l}\text { Categoría Disponibilidad para la } \\
\text { atención }\end{array}$ & 0,108 & 0,122 & & $\begin{array}{l}\text { Categoría Disponibilidad para la } \\
\text { atención }\end{array}$ & 0,777 & 0,792 \\
\hline \multirow{10}{*}{ 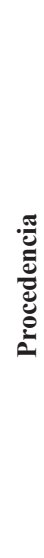 } & Resultados global & 0,053 & 0,053 & \multirow{10}{*}{ 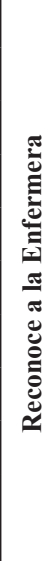 } & Resultados global & 0,225 & 0,212 \\
\hline & Categoría Sentimientos del paciente & 0,105 & 0,099 & & Categoría Sentimientos del paciente & 0,056 & 0,046 \\
\hline & $\begin{array}{l}\text { Categoría Características de la } \\
\text { enfermera }\end{array}$ & 0,160 & 0,160 & & $\begin{array}{l}\text { Categoría Características de la } \\
\text { enfermera }\end{array}$ & 0,592 & 0,548 \\
\hline & Categoría Dar apoyo emocional & 0,169 & 0,178 & & Categoría Dar apoyo emocional & 0,710 & 0,654 \\
\hline & Categoría Dar apoyo físico & 0,091 & 0,077 & & Categoría Dar apoyo físico & 0,126 & 0,137 \\
\hline & $\begin{array}{l}\text { Categoría Cualidades del hacer de la } \\
\text { enfermera }\end{array}$ & 0,030 & 0,029 & & $\begin{array}{l}\text { Categoría Cualidades del hacer de la } \\
\text { enfermera }\end{array}$ & 0,051 & 0,061 \\
\hline & Categoría Proactividad & 0,403 & 0,434 & & Categoría Proactividad & 0,080 & 0,065 \\
\hline & Categoría Empatía & 0,305 & 0,313 & & Categoría Empatía & 0,674 & 0,669 \\
\hline & Categoría Priorizar al ser de cuidado & 0,457 & 0,442 & & Categoría Priorizar al ser de cuidado & 0,284 & 0,274 \\
\hline & $\begin{array}{l}\text { Categoría Disponibilidad para la } \\
\text { atención }\end{array}$ & 0,324 & 0,304 & & $\begin{array}{l}\text { Categoría Disponibilidad para la } \\
\text { atención }\end{array}$ & 0,020 & 0,023 \\
\hline \multirow{10}{*}{ 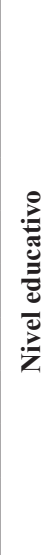 } & Resultados global & 0,029 & 0,101 & \multirow{10}{*}{ 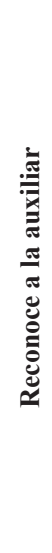 } & Resultados global & 0,069 & 0,073 \\
\hline & Categoría Sentimientos del paciente & 0,123 & 0,118 & & Categoría Sentimientos del paciente & 0,477 & 0,477 \\
\hline & $\begin{array}{l}\text { Categoría Características de la } \\
\text { enfermera }\end{array}$ & 0,000 & 0,001 & & $\begin{array}{l}\text { Categoría Características de la } \\
\text { enfermera }\end{array}$ & 0,741 & 0,527 \\
\hline & Categoría Dar apoyo emocional & 0,041 & 0,101 & & Categoría Dar apoyo emocional & 0,692 & 0,682 \\
\hline & Categoría Dar apoyo físico & 0,136 & 0,107 & & Categoría Dar apoyo físico & 0,055 & 0,076 \\
\hline & $\begin{array}{l}\text { Categoría Cualidades del hacer de la } \\
\text { enfermera }\end{array}$ & 0,472 & 0,415 & & $\begin{array}{l}\text { Categoría Cualidades del hacer de la } \\
\text { enfermera }\end{array}$ & 0,026 & 0,027 \\
\hline & Categoría Proactividad & 0,190 & 0,281 & & Categoría Proactividad & 0,003 & 0,003 \\
\hline & Categoría Empatía & 0,519 & 0,407 & & Categoría Empatía & 0,828 & 0,855 \\
\hline & Categoría Priorizar al ser de cuidado & 0,211 & 0,238 & & Categoría Priorizar al ser de cuidado & 0,091 & 0,093 \\
\hline & $\begin{array}{l}\text { Categoría Disponibilidad para la } \\
\text { atención }\end{array}$ & 0,722 & 0,710 & & $\begin{array}{l}\text { Categoría Disponibilidad para la } \\
\text { atención }\end{array}$ & 0,176 & 0,205 \\
\hline
\end{tabular}

Fuente: PCHE, 2015. 


\section{DISCUSIÓN}

Diariamente, el personal de enfermería se ve enfrentado a diferentes situaciones que se centran en las vivencias de los pacientes a quienes se les brinda cuidado. Por diversas circunstancias, no se tiene la posibilidad de un momento de retroalimentación entre paciente - enfermera(o) que facilite el crecimiento de esta interacción y el fortalecimiento del cuidado que se brinda, teniendo en cuenta que la esencia de enfermería son las relaciones interpersonales, las cuales involucran una serie de contactos tanto verbales como también gestos y movimientos, entre otros, que dan como resultado la percepción del sujeto de cuidado que determina la calidad de la atención que ofrece el personal de enfermería (22).

Al comparar los resultados encontrados en esta investigación con otros estudios realizados en Colombia $(1,14-15,23)$ que abordaron el tema de percepciones de cuidado en centros hospitalarios, se encontró que las participantes perciben el comportamiento humanizado por enfermería, de manera global, entre excelente y bueno, lo cual indica que el personal está calificado para brindar una atención con calidad y calidez; sin embargo, se debe evaluar constantemente la atención, para así disminuir e identificar las fallas que hacen que la percepción de los sujetos de cuidado sea inconforme.

De la experiencia ofrecida por el personal de enfermería durante este proceso de cuidado, un aspecto positivo enmarca a tres categorías que lograron los mejores resultados: características de la enfermera, seguido de priorizar al ser de cuidado y, en tercer lugar, proactividad, lo que permite resaltar la importancia de adquirir habilidades y actitudes fundamentales a la hora de iniciar una relación de ayuda con los sujetos de cuidado.

La categoría características de la enfermera, descrita en el estudio de Rivera (10), reveló resultados sobresalientes que relacionan a la enfermera(o) con aspectos de una persona cálida, agradable, amable y cordial; cualidades perceptibles en la interacción durante las actividades de cuidado y que facilita el tratamiento. Vidal (24) menciona que "la calidad de la comunicación de la enfermera disminuye la ansiedad, mejora sus habilidades de autocura y consigue una mayor adherencia al tratamiento"
La categoría priorizar el ser de cuidado revela resultados similares en estudios análogos dentro de los cuales González (25) destaca un aspecto importante afirmando que "priorizar el cuidado hacia el paciente y la familia debe convertirse en el eje central del trabajo de los profesionales de enfermería, haciendo que los procedimientos sean vistos como prioridad hacia el paciente y no prioridad para cumplir funciones emanadas por otros profesionales", razón que destaca la importancia del enfoque principal del cuidado y que, por su mención en el estudio, revela un posible cambio en la prioridades del cuidado de enfermería.

La categoría de proactividad forma una alianza terapéutica entre la (el) enfermera(o) y el sujeto de cuidado, en la cual se genera, de cierta manera, una correlación especial, evidenciada en el estudio de González (15), en donde esta categoría destaca la buena actitud frente a las necesidades de los pacientes, la respuesta oportuna a sus llamados, responder las dudas de las gestantes e infundir el llamado ante cualquier eventualidad como aspectos que integran ese conglomerado de procesos importantes del cuidado humanizado y que deben considerarse como elementos significativos para que se expresen y faciliten los momentos de interacción (26).

Otra circunstancia determina las categorías que alcanzaron una menor frecuencia de ser percibidas por la población encuestada, de las cuales cabe destacar dar apoyo emocional y empatía que, como se mencionó anteriormente, son cualidades que se perciben mediante la interacción con el otro y que exigen cierto grado de confianza, condición de esto, el tiempo requerido para establecer comunicación en la interacción enfermera-paciente y que, en la práctica, encuentra algunas dificultades entre las exigencias del servicio y el personal disponible, evidenciado en el número de pacientes que ingresan al servicio anualmente, generando, por tanto, un déficit en la calidad del cuidado humanizado.

Ese déficit está relacionado con la comprensión, el respeto, la dedicación, la confianza y la comunicación, aspecto que obtuvo una calificación entre deficiente y aceptable en la percepción encontrada por la mayoría de las gestantes, quienes estaban en el nivel de educación secundaria y superior, posición evidenciada por algunos comentarios de los sujetos de cuidado:
ISSN 1794-9831

E-ISSN 2322-7028

Vol. 13 No. 2

Jul - Dic 2016

Cúcuta, Colombia 
E-ISSN 2322-7028

Vol. 13 No. 2

Jul - Dic 2016

Cúcuta, Colombia
"Muchas veces ingresan a la habitación, no saludan, ni a mí ni a mi familiar. Entran y salen como si no estuviéramos aquí..." G87†.

"De antemano sé que deben estar cansadas por todas las pacientes que atienden, pero eso no significa que no sean amables o respetuosas al hablarme, especificamente cuando les pregunto algo, no generan un debido ambiente de comunicación" G94".

Este aspecto cobra especial interés en la atención de enfermería, la identificación del tipo de amabilidad, la comunicación, la dedicación del cuidado hacia las gestantes, lo que abarca un eje importante de la relación interpersonal de Watson (27), quien plantea en su teoría fundamental que "el contacto entre dos seres humanos puede ayudar a los enfermos a descubrir nuevos conocimientos que ayuden con la recuperación y promover la armonía" para un cuidado humanizado, así como también la compañía y presencia del personal médico, la generación de confianza y tranquilidad mezcladas con amor y dedicación, permitiendo satisfacer las necesidades de las usuarias en el proceso de gestación.

Son los profesionales de enfermería, por su formación en el arte de cuidar, quienes establecen la comunicación entre el personal de salud y el sujeto de cuidado; ellos son quienes tienen la capacidad de relacionarse con los sentimientos y las emociones y quienes perciben, mediante los gestos verbales y no verbales, el estado de ánimo de la persona. La empatía es muy importante para la profesión como habilidad comunicativa y desarrollo personal, puesto que, a través de ella, se establece una interacción y un vínculo de humanización que le permite al sujeto expresar sus alegrías y temores, haciendo más llevadera su dolencia y ayudando en la recuperación física y mental (28).

La empatía, como ítem, obtuvo el menor valor en cuanto a las percepciones de cuidado humanizado manifestadas por las gestantes, evidenciando que el vínculo de atención entre enfermera y paciente es deficiente; por lo tanto, se propone para el personal de enfermería la necesidad de fortalecer las habilidades para la vida, enfatizando en la comunicación y afectividad, que son herramientas indispensables para la atención de la gestante y, en general, del cuidado de la persona a nivel intrahospitalario o ambulatorio.

\footnotetext{
$\dagger$ G87: gestante 87 .

\$ G94: gestante 94 .
}

González (15) obtuvo resultados similares en su estudio, encontrando que esta categoría alcanzó el promedio más bajo. Por otro lado, Gonsalves (29) describe, en su artículo, el uso de la empatía en el equipo de enfermería: "Se observa que la preocupación de proporcionar atención a los pacientes de una manera humana y el medio ambiente de trabajo pueden generar estrés y malestar, siendo la interacción de la empatía en la relación entre personal de enfermería y sujeto de cuidado, la que hace que la misma no se torne fría e indiferente"(29).

El estudio demostró que el grado empatía-pacienteenfermera no es el adecuado, por cuanto en la mayoría de casos manifestados por las gestantes no se tenía ningún contacto cercano con la enfermera (o) de turno, haciendo que no existiera una relación cercana ya que, en general, el personal de enfermería referido no contaba con el tiempo suficiente para escuchar detenidamente aquello que aqueja a la paciente, más que significar una dolencia superficial. Este hecho fue constatado mediante una observación directa al momento de realizar la encuesta a las maternas.

No cabe duda que en los comentarios de las gestantes se reflejó lo mencionado anteriormente. Una paciente lo relata de la siguiente forma:

"Ellas se dedican a hacer el trabajo que les toca, por el que les pagan, colocar la droga, tomar la presión; pero son contadas las veces que preguntan ¿usted está triste?, ¿tiene alguna duda?, si quiere puede hablar conmigo" G21".

Se necesita interés por parte del profesional, en la práctica, donde se evidencie el ejercicio continuo y desarrollo de esta competencia interpersonal, que se caracteriza por ser uno de los elementos básicos y necesarios para trabajar efectivamente con el otro, en una relación de ayuda, según lo manifestado por Rogers (30). Por otro lado, para Jean Watson (27), cuando el profesional de enfermería es empático, logra captar con claridad el mundo del paciente y puede moverse libremente dentro de él.

A lo largo del estudio se identifica que hace falta la interiorización y proyección de la empatía en el profesional de la institución. Así mismo, es necesaria la capacidad para pensar y sentir el mundo interno del
I G21: gestante 21 . 
paciente, permitiendo experimentar los sentimientos de la otra persona sin perder la capacidad de evaluar objetivamente los estados psicosociales del otro.

La encuesta enmarca, dentro del perfil sociodemográfico, características que influyen significativamente en la percepción de las pacientes. Aunque dichos resultados no han sido valorados en anteriores estudios del mismo instrumento -y no aparecen como específicos de la herramienta PCHE, se pudo encontrar una relación significativa (tabla 3) según correlación estadística, mediante pruebas de Pearson y Fisher.

Estas correlaciones estadísticas ponen de manifiesto variables a tener en cuenta como procedencia, nivel educativo y estrato, además de las categorías que generaron mayor significancia estadística y que, en relación con el profesional de enfermería, destacan elementos que miden rasgos distintivos propios de la profesión y que implican la convergencia de conocimientos y vocación, medidos mediante las categorías de características de la enfermera y cualidades del hacer de la enfermera.

Al respecto, conviene decir que se reconocen características que están sujetas a las pacientes y que modifican, en cierto grado, la percepción del cuidado. Dicho esto, se destaca la interpretación de los ítems “¿reconoce a la enfermera(o)?” y “¿reconoce a la auxiliar?" como elementos importantes que, si bien individualmente sólo describen el interés por parte de las gestantes y el personal de salud por un mutuo reconocimiento, en conjunto son los elementos que conforman las categorías.

Adicionalmente, se resalta el valor de la comunicación en el enfoque de la calidad del cuidado, que se expresa mediante los resultados positivos cuando existió una comunicación mutua lo suficientemente significativa como para destacar el valor del cuidado prestado por un enfermero o auxiliar.

De esta manera, se puede resaltar que el cuidado humanizado es indispensable para la atención de las gestantes, donde enfermería entra a jugar un papel muy importante ya que es quien idealmente se encuentra en contacto directo y continuo con los sujetos de cuidado, exigiendo del profesional una amplia preparación técnica, científica y humana para brindar una atención de calidad, características que deben estar enmarcadas por la vocación de servicio (31).
De acuerdo con Cantillo (32), el cuidado brindado por el personal de enfermería del hospital es humanizado y se caracteriza por el respeto, la eficiencia, la claridad en la comunicación, la solidaridad, el compromiso, el conocimiento, el desarrollo de habilidades profesionales, el trabajo en equipo y el liderazgo, la seguridad, la atención en las necesidades básicas y la búsqueda permanente de la excelencia del cuidado. Esto implica que para que el personal de enfermería pueda ejecutar el cuidado, su objeto de conocimiento y razón de ser requiere de una formación científica, tecnológica y humanística que le permita, en su interacción con los pacientes, realizar acciones que exigen la participación de ambos.

Ahora bien, si los pacientes no tienen las condiciones necesarias para la interrelación eficaz con el entorno y con las enfermeras, se requiere que éstas propongan alternativas de cuidado mediante una sólida base de conocimientos científicos y habilidades prácticas y con un alto grado de sensibilidad humana, para interpretar adecuadamente, mediante la valoración, la escucha y la relación con los familiares, la condición de la paciente.

\section{CONCLUSIONES}

El resultado global de comportamiento de cuidado humanizado de enfermería en todas las categorías fue calificado como bueno y excelente, lo que demuestra que existe una adecuada percepción por parte de las gestantes y demuestra que el personal de enfermería está guiándose por la ruta adecuada de humanización del cuidado en sus actividades diarias

Las categorías de mayor porcentaje fueron: características de la enfermera, priorizar al ser cuidado y proactividad, por lo cual es importante fortalecer estos aspectos para que el personal posea capacidades y conocimientos requeridos para seguir brindando un cuidado humanizado oportuno y adecuado a cada usuario que atiende en el servicio.

Cualquier sistema de salud -aparte de ser moderno, competitivo y comprometido con las actuales características que rigen una atención de calidad en salud- debe tener en cuenta las características del personal a la hora de la atención, aspecto que toma relevancia para el personal de enfermería del hospital, demostrando calidad, simpatía y amabilidad, así como cordialidad en la atención humanizada, fenómeno 
ISSN 1794-9831

E-ISSN 2322-7028

Vol. 13 No. 2

Jul - Dic 2016

Cúcuta, Colombia evidenciado en los resultados con mayor porcentaje.

Las categorías con más bajo valor fueron empatía y disponibilidad para la atención, considerando que estas acciones son claves fundamentales a la hora de brindar un cuidado humanizado. Por ello, se hace necesario analizar y estudiar qué circunstancias impiden que haya una empatía y disponibilidad por parte de las enfermeras y establecer un plan de atención para la mejoría de estas acciones en pro de otorgar un excelente cuidado humanizado.

las gestantes en mejor posición económica, educativa y de procedencia manifiestan, en concordancia con los resultados obtenidos, una mayor exigencia a la hora de evaluar comportamientos relacionados con estas categorías en comparación con las demás gestantes encuestadas y que implicaría una relación directa entre conocimiento de las participantes y la percepción del cuidado.

La humanización del cuidado es un elemento que distingue a los profesionales de enfermería del resto del personal de salud, lo que establece la invitación a incluir con mayor rigor la práctica humanística en la profesión e integración de la misma en los actores de la familia y la comunidad.

\section{CONFLICTO DE INTERESES}

Los autores declaran no tener ningún conflicto de intereses. 


\section{REFERENCIAS BIBLIOGRÁFICAS}

1. Poblete-Troncoso M, Valenzuela-Suazo S. Cuidado humanizado: un desafío para las enfermeras en los servicios hospitalarios. Acta Paul Enferm. 2007;20(4): 499-503.

2. Muñoz-Hernández Y, Coral-Ibarra R, Moreno-Prieto D, Pinilla-Pinto D, Suárez-Rodríguez Y. Significado del cuidado humanizado en egresadas de la facultad de enfermería. Repertorio de medicina y cirugía. 2009; 18(4): 246-50.

3. Martínez FT. Enfermería y las ciencias sociales; un enfoque diferente o una necesidad para entender el cuidado. Enf Neurol (Mex). 2011; 10(2): 110-3.

4. Baêta-Neves CA. Saúde: a cartografia do trabalho vivo. Cadernos de Saúde pública. 2008;24(8):1953-5.

5. Urra E, Jana A, García M. Algunos aspectos esenciales del pensamiento de Jean Watson y su teoría de cuidados transpersonales. Ciencia y enfermería. 2011; 17(3): 11-22.

6. Ávila-Urdaneta M. La Salud en el paradigma de la Descentralización en América Latina. Revista de Ciencias Sociales. 2010; 16(3): 526-43.

7. Martins JdJ, Backes DS, Cardoso RdS, Erdmann AL, Albuquerque GLd. Resignificando la humanización desde el cuidado en el curso de vivir humano. Rev enferm UERJ. 2008; 16(2): 276-81.

8. Ruydiaz-Gómez KS, Fernández-Aragón SP. Incertidumbre y apoyo social en gestantes con trastornos hipertensivos. Index de Enfermería. 2015; 24(1-2): 67-70.

9. Silva-Fhon J, Ramón-Cordova S, Vergaray-Villanueva S, Palacios-Fhon V, Partezani-Rodrigues R. Percepción del paciente hospitalizado respecto a la atención de enfermería en un hospital público. Enfermería Universitaria. 2015; 12(2): 80-7.

10. Rivera LN, Triana Á. Percepción de comportamientos de cuidado humanizado de enfermería en la Clinica del Country. Av enferm. 2007; 25(1):56-68.

11. Reina-Gamba RC, Vargas-Rosero E. Validez de contenido y validez facial del instrumento "Percepción de comportamientos de cuidado humanizado". Av enferm. 2008; 26(2): 71-9.

12. Onsalus.com. Definición de apoyo emocional. [Internet]. 2014 [consultado 12 de octubre de 2014]. Disponible en: http://www.onsalus.com/index.php/diccionario/apoyo-emocional/1851.

13. Contreras-Rojas EG. Precisando el concepto de apoyo emocional en el que hacer de enfermería. Servicio de Medicina Interna 7aB. Hospital Daniel Alcides Carrión, 2007 [Tesis]. Perú: Universidad Nacional Mayor de San Marcos; 2008.

14. Ceballos-Vásquez PA. Desde los ámbitos de enfermería analizando el cuidado humanizado. Ciencia y Enfermería. 2010; 16(1): 31-5.

15. Gonzalez-Burgos JT, Quintero-Martinez DC. Percepción del cuidado humanizado en pacientes que ingresan al servicio de hospitalizacion de urgencias de ginecobstetricia de una institucion de II y III nivel de atención [Tesis]. Colombia: Pontificia Universidad Javeriana; 2009.

16. Molano-Pirazán ML, Guerrero NS. Características del enfermero que cuida y de la persona cuidada en un ambiente neonatal. Rev Cubana de Enfermer. 2012; 28(2): 169-80.

17. Hernández G. Que es cuidado de enfermería. Grupo de Cuidado UDES [Internet]. 2013 [consultado 12 de agosto de 2014]. Disponible en: http://grupodecuidadoudes.blogspot.com.co/2013/04/que-es-cuidado-de-enfermeriapor-gloria.html

18. Landman C, Osorio C, Jesús M, García-García E, Pérez-Meza P, Sandoval-Barrera P, et al. Satisfacción usuaria respecto a competencia de comunicación del profesional de enfermería. Ciencia y Enfermería. 2015; 21(1): 91102.

19. Marroquín-Peña R. Confiabilidad y validez de intrumentos de investigación [Internet]. Perú: Universidad Nacional de Educación Enrique Guzmán y Valle 2013 [consultado 22 de octubre de 2014]. Disponible en: http://www.une.edu.pe/Titulacion/2013/exposicion/SESION-4-Confiabilidad y Validez de Instrumentos de investigacion.pdf.

20. Arias-Pittman JA. Confiabilidad y validez para evaluar los instrumentos de medición: Aplicacion en SPSS y EXCEL [Tesis de Maestria]. Perú: Universidad Nacional José Faustino Sánchez Carrion; 2013.

21. República de Colombia, Ministerio de Salud. Resolución No. 008430 de 1993, octubre 4, por la cual se establecen las normas científicas, técnicas y administrativas para la investigación en salud. Bogota D.C.: Ministerio de Salud; 1993.

22. Ramírez P, Müggenburg C. Relaciones personales entre la enfermera y el paciente. Enfermería Universitaria. 2015; 12(3): 134-43.

23. Bautista-Rodríguez LM, Parra-Carrillo EL, Arias-Torres KM, Parada-Ortiz KJ, Ascanio-Meza KA, Villamarin- 
Capacho MI, et al. Percepción de los comportamientos de cuidado humanizado en los usuarios hospitalizados en una institución de salud de $3^{\circ}$ y $4^{\circ}$ nivel de atención. Rev. cienc. cuidad. 2015; 12(1): 105-118.

24. Vidal-Blan R, Adamuz-Tomás J, Feliu-Baute P. Relación terapéutica: el pilar de la profesión enfermera. Enfermería Global. 2009; 1(17): 0-0.

25. González-Ruiz G, Oviedo-Córdoba H, Bedoya-Idrobo LM, Benavides-Peñaloza G, Dalmero-Arvilla N, VergaraAscanio C. Cuidado humanizado de enfermería percibido por familiares de pacientes pediátricos, usuarios del Hospital Universitario Fernando Troconis de Santa Marta. Memorias. 2013; 11(20): 31-9.

26. Chernicharo IM, Duarte F , Ferreira M. Description of the term humanization in care by nursing professionals. Escola Anna Nery. 2014; 18(1): 156-62.

27. Watson J. Watson s theory of human caring and subjective living experiences: carative factors/caritas processes as a disciplinary guide to the professional nursing practice. Texto Contexto -enferm. 2007; 16(1): 129-35.

28. Landete-Belda L. La comunicación: Pieza clave en Enfermería. Enfermería dermatológica 2012 May- Ago; (16): 16-19.

29. Gonsalves T, Pintanela D, Rodrigues L, Silva CF, Silva R, Farias G, et al. O uso da empatia por profissionais de enfermagem: uma revisão integrativa. En: $12^{\mathrm{a}}$ Mostra de Produção Universitària. Congresso de Iniciação Científica. Rio Grande R/S, Brasil; 23 a 25 de outubro de 2013.

30. Rogers CR. The necessary and sufficient conditions of therapeutic personality change. Journal of Consulting and Clinical Psychology. 1992; 60(6): 827.

31. Armijos-Erazo N, Chamba-Pullaguari JL. El Área Laboral como espacio profesional de los y las Trabajadoras Sociales en la defensa de los derechos y deberes de los trabajadores de PREDESUR en la Ciudad de Loja [Tesis]. Ecuador: Universidad Nacional de Loja; 2011.

32. Cantillo EV. Seguridad de los pacientes: Un compromiso de todos para un cuidado de calidad. Salud Uninorte. 2007; 23(1): 112-9. 